\title{
Preparation of Ziegler-Natta Catalysts for the Synthesis of Polypropylene/Carbon Nanotubes Nanocomposites by in situ Polymerization
}

\author{
Renato Jonas Benne de Oliveira, Juliana da Silva Santos, Maria de Fátima Vieira Marques \\ Professora Eloisa Mano Institute of Macromolecules - IMA, Rio de Janeiro Federal University - UFRJ
}

\begin{abstract}
Aiming to improve the dispersion of carbon nanotubes (CNT) in a polymer matrix, a fourth-generation Ziegler-Natta, ZN, catalyst was prepared in the presence of CNT to synthesize nanocomposites of polypropylene (PP/CNT) by in situ polymerization. The performance of the ZN catalyst was evaluated along with the thermal (TGA and DSC) and dynamic-mechanical properties of the nanocomposites and they were compared with those of neat PP synthesized with the standard catalyst prepared without carbon nanoparticles. The thermal degradation temperature of the PP/CNT nanocomposite increased in comparison with that of neat PP. Moreover, the crystallization temperature and the degree of crystallinity sharply increased in PP/CNT materials. Finally, the storage modulus of the nanocomposite prepared by $30 \mathrm{~min}$ of polymerization increased, as well as the $\mathrm{Tg}$.
\end{abstract}

Keywords: Polypropylene, nanocomposite, in situ polymerization, carbon nanotubes, Ziegler-Natta.

\section{Introduction}

Nanotechnology has been widely studied to develop methods to enhance the properties and find new applications for polymers ${ }^{[1]}$. The search for polymer materials with superior properties has been the subject of numerous studies ${ }^{[2,3]}$. Materials with superior resistance in relation to conventional composites can be prepared by combining a soft polymer matrix with rigid nanoparticles as fillers ${ }^{[4]}$.

CNTs have been widely used as reinforcement to produce polymer materials with interesting and novel properties ${ }^{[5,6]}$. These have a structure similar to a graphene sheet rolled into a transparent cylinder and can be classified as single-walled or multi-walled carbon nanotubes ${ }^{[7]}$.

To obtain PP nanocomposites with CNTs with optimum properties, the nanofillers should be well dispersed without agglomerations and distributed homogeneously in the polymer matrix. Poor distribution can cause stress concentration and act negatively on the material's properties. If the dispersion is not appropriate, the contact between the nanofillers with high specific area and the matrix is compromised, and the aggregates of nanofillers can act as defects in the material ${ }^{[8]}$.

The preparation of nanocomposites involves the appropriate choice of a method to achieve satisfactory dispersion of nanofillers through the polymer matrix. The in situ polymerization technique is an effective method which provides good dispersion of nanofillers in the polymer matrix ${ }^{[9]}$. In the case of PP, this method comprises the deposit of the active sites on the nanofillers' surface to compose the solid catalyst, and during polymerization the polymer chains grow on the nanofiller's surface.

Funck and Kaminsky ${ }^{[10]}$ produced PP nanocomposites with multi-walled carbon nanotubes (MWCNT) by in situ polymerization using a metallocene catalyst based on zirconium. By oxidation in a solution of $\mathrm{H}_{2} \mathrm{SO}_{4}: \mathrm{HNO}_{3}$, hydroxyl and carboxyl groups were inserted on the walls of MWCNT to covalently attach them to the cocatalyst methylaluminoxane, and after polymerization, the formation of materials with fillers well dispersed throughout PP matrix could be observed.

In the preparation of nanocomposites by in situ polymerization, the monomer is polymerized in the presence of a nanofiller, which provides better contact between the polymer and the dispersed phase ${ }^{[11]}$. In situ polymerization allows the mixing of nanofillers in the polymer matrix during the birth of the polymer, thus eliminating a later step of mixing, resulting in time saving and reduced production cost. Furthermore, this technique allows the preparation of nanocomposites with high concentrations of filler without compromising the polymer matrix.

Ziegler-Natta catalysts are the most used systems in industry for the production of polyolefins ${ }^{[12,13]}$. Generally, a Ziegler-Natta catalyst is a complex formed by the reaction of transition metal compounds (derivative of alkyl, aryl of alkoxide or halide) of groups IV to VIII of the periodic table, with an alkyl metal or alkyl-metal halide of groups I to III. Compounds of transition metals containing titanium, vanadium, chromium and, in special cases, molybdenum, cobalt, rhodium and nickel are the most used ${ }^{[14-16]}$

For the isospecific polymerization of propylene from a latest-generation Ziegler-Natta, a titanium compound supported on magnesium chloride is employed and internal electron donors (introduced into the solid catalyst) and external ones (inserted together with the cocatalyst in the polymerization) are added to increase the kinetics as well as stereochemistry of the catalys $\mathrm{t}^{[12,14,17]}$. An 
interesting feature of such heterogeneous Ziegler-Natta systems is their control of polymer particle morphology in spherical shape. Thus, the synthesized polymer tends to replicate the morphology of the catalyst, providing the synthesis of PP already in the form of pellets, which can eliminate a subsequent production step of extrusion for pelletization $^{[18]}$.

Therefore, the aim of this work was to prepare an isospecific Ziegler-Natta catalyst based on $\mathrm{MgCl}_{2} / \mathrm{TiCl}_{4}$ in the shape of spherical particles containing carbon nanotubes in order to polymerize propylene and thus obtain a PP/CNT nanocomposite with good dispersion of fillers in the matrix and to evaluate the thermal and dynamic-mechanical properties of the polymer materials obtained.

\section{Methods}

\section{Preparation of standard adduct and adduct containing CNTS}

In a reactor, $80 \mathrm{~mL}$ of mineral oil was added under inert atmosphere. Then $3 \mathrm{~g}$ of anhydrous $\mathrm{MgCl}_{2}$ was added and after 5 minutes, anhydrous ethanol was introduced to form the alcoholic adduct at molar ratio $1 \mathrm{MgCl}_{2}: 3$ ethanol. After 15 minutes, the temperature was raised over 3 hours to the melting of the adduct. The molten material was transferred to a flask containing isoparaffin at $-40{ }^{\circ} \mathrm{C}$. After precipitation, the supernatant was removed and the precursor of the catalytic support was washed and dried ${ }^{[19]}$.

The preparation of the catalyst support precursor was carried out similarly to the standard precursor, differing only by the addition of carbon nanotubes $\left(2 \mathrm{MgCl}_{2}: 1 \mathrm{CNT}\right.$, wt.), which were homogenized by ultrasonic bath before the addition of $\mathrm{MgCl}_{2}$ and ethanol. Multi-walled carbon nanotubes - FeCoMgO $\sim 95 \%$ carbon were used.

\section{Dealcoholization with titanium tetrachloride and preparation of catalyst}

$\mathrm{TiCl}_{4}$ used for the dealcoholization at molar ratio $1 / 2 \mathrm{TiCl}_{4}: 1 \mathrm{EtOH}$. $\mathrm{TiCl}_{4}$ in isoparaffin was added to the homogenized adduct in at $0{ }^{\circ} \mathrm{C}$ and $250 \mathrm{rpm}$. After that, the temperature was raised to $60{ }^{\circ} \mathrm{C}$ remaining for 4 hours $^{[20]}$. Then the supernatant was removed and the support was washed at $60{ }^{\circ} \mathrm{C}$ with hexane.

To the catalytic support, $15 \mathrm{~mL}$ of isoparaffin was added and the internal electron donor (DI) n-butyl phthalate was introduced at $60{ }^{\circ} \mathrm{C}$. The molar ratio used was $8 \mathrm{MgCl}_{2}: 1 \mathrm{DI}$. The temperature was raised to $100^{\circ} \mathrm{C}$ at $400 \mathrm{rpm}$ and maintained for 2 hours. Subsequently, $10 \mathrm{ml}$ of $\mathrm{TiCl}_{4}$ was added, the system was heated to $120^{\circ} \mathrm{C}$ and held for 2 hours. Next, the supernatant was removed and the catalytic solid was washed with dry hexane at $60{ }^{\circ} \mathrm{C}$ and dried until constant mass ${ }^{[18]}$.

\section{Polymerization}

Polymerizations were carried out in Büchi reactor with mechanical stirrer $(650 \mathrm{rpm})$ and 1-liter jacket glass beaker connected to a Haake model N6 thermostatic bath. The glass reactor was dried at $120^{\circ} \mathrm{C}$ and was fixed on the metal support under flowing nitrogen. After cooling, $80 \mathrm{ml}$ of hexane, the cocatalyst triethylaluminium (TEA) and external donor (diphenyl-dimethoxysilane) were introduced under stirring. $0.1 \mathrm{~g}$ of solid catalyst was added, propylene was introduced and pressure was raised to 4 bar and maintained at $70{ }^{\circ} \mathrm{C}^{[21]}$. Polymerization was conducted until the desired reaction time. The polymers obtained were poured into a beaker with $5 \% \mathrm{HCl} / \mathrm{ethanol}$ and kept under stirring overnight. After this period, the polymer was washed, vacuum filtered and kept in an oven at $60{ }^{\circ} \mathrm{C}$ until constant mass ${ }^{[19]}$.

\section{Characterization}

The titanium content was measured by energy dispersive X-ray spectrometry in a Shimadzu model 720 EDX apparatus. Previously dried samples of ZieglerNatta catalysts were examined under vacuum for 320 seconds in powdered form ${ }^{[22]}$.

Scanning electron microscopy was employed to characterize the catalysts and the surfaces of the specimens of polymers. The samples were cryo-fractured in order to maintain the morphological structure of the polymers $^{[23]}$.

The thermal stability of the polymer materials was measured by thermogravimetry (TGA) using a TA Instruments model Q 500 thermal analyzer. $\mathrm{T}_{\text {onset }}$ represents the start of degradation and $\mathrm{T}_{\max }$ is the temperature of maximum degradation rate, observed as a peak of the derivative ${ }^{[24]}$. Samples were heated from room temperature to $700{ }^{\circ} \mathrm{C}$ at a heating rate of $10{ }^{\circ} \mathrm{C} / \mathrm{min}$ under an inert atmosphere of $\mathrm{N}_{2}$.

Transmission electron microscopy (TEM) was used to analyze the morphology of CNTs and their distribution in the polymer matrix ${ }^{[25]}$.

For the DSC analysis, about $4.5 \mathrm{mg}$ of the sample was used. The polymer was heated from room temperature to $180{ }^{\circ} \mathrm{C}$ at a rate of $10{ }^{\circ} \mathrm{C} / \mathrm{min}$ and then cooled to $25^{\circ} \mathrm{C}$. Again, a TA Instruments model Q 1000 analyzer was employed $^{[18]}$.

For the dynamic-mechanical analysis, rectangular samples $(18 \times 14 \times 2 \mathrm{~mm})$ were obtained by pressing at $200{ }^{\circ} \mathrm{C}$ for $10 \mathrm{~min}$ with subsequent conditioning at $80^{\circ} \mathrm{C}$ for $48 \mathrm{~h}$. The storage (E') and loss (E") moduli were evaluated, as well as the glass transition temperature of the materials ${ }^{[26]}$. A TA Instruments DMA Q800 thermodynamic-mechanical analyzer was used with single cantilever mode at a frequency of $1 \mathrm{~Hz}, 0.1 \%$ deformation and heating rate of $2{ }^{\circ} \mathrm{C} / \mathrm{min}$.

\section{Results and Discussion}

Ziegler-Natta catalysts based on titanium species supported on magnesium chloride were prepared by the emulsion technique. All steps of the catalyst preparation were controlled to obtain the catalyst with spherical shape so that the polymer replicated the nature of the catalyst. For comparison, a standard Ziegler-Natta catalyst (CAT-1) was also prepared and contrasted with the Ziegler-Natta catalyst containing carbon nanotubes immobilized on its structure (CAT-CNT). For the preparation of the CATCNT, a mass ratio of 1:1 between the magnesium chloride 
and CNTs was used. The morphology of the CNTs and the catalysts was evaluated by scanning electron microscopy (SEM) micrographs, as shown in Figure 1.

The morphology of tangled wires typical of CNTs is shown in Figure 1a. Although both catalysts presented globular morphology, as shown in Figure 1b-c, there was a change in the aspect of the catalyst containing CATCNT carbon nanotubes compared to the standard catalyst CAT-1. The CAT-CNT (Figure 1c) showed an increase in porosity of the surface, which can be attributed to the presence of carbon nanotubes. Furthermore, the image of CAT-CNT showed that carbon nanotubes were involved by the catalytic system, which would allow the polymer during its synthesis to grow in between the meshes of the CNT, providing the disentanglement of carbon nanotubes.

The titanium content of the catalysts was determined by EDX. CAT- 1 showed a titanium content of $7.1 \% \mathrm{~m} / \mathrm{m}$ while the CAT-CNT contained only $5.4 \%$, which shows that the titanium sites were probably not fixed directly to the surface of the nanotube, but mostly through the support magnesium chloride.

Table 1 shows the yield of polymerizations conducted at different reaction times. Within $60 \mathrm{~min}$ of polymerization, the catalyst CAT-CNT produced a higher yield compared to standard CAT-1. It would be expected that a higher titanium content of the catalyst would lead to polymerizations with higher yield, though a part of the titanium can be found as inactive form. The higher polymer yield obtained with the catalyst containing CNT may be related to its greater porosity, since the titanium content of CAT-CNT was lower than that of the CAT-1.

It can be also noted that the yields of reactions with CAT-CNT for $15 \mathrm{~min}$ and $30 \mathrm{~min}$ were much lower than the reaction for $60 \mathrm{~min}$. This may be due to diffusional hindrance of movement of the monomer to the active center in the catalyst with CNT. The fragmentation of the catalyst could be also hindered by the entanglements of the nanotubes. After fragmentation of the catalyst particles during synthesis of the polymer, yield rises rapidly.

By thermogravimetry it was possible to evaluate the thermal stability of the materials as well as to estimate the amount of CNT in PP. From the mass loss, it was found that the PP-1 completely decomposed up to $700{ }^{\circ} \mathrm{C}$, leaving only a small fraction of the mass, which was attributed to catalytic residue. The PP1/CNT lost $98.8 \%$ of its mass. This might be related to the high yield of this polymerization, leading to a high dilution of nanofiller in the polymer matrix. In fact, only around $33 \mathrm{mg}$ of CNT was added in each polymerization and according to the yield obtained at $60 \mathrm{~min}$ of reaction, the CNT content is $c a .0 .5 \% \mathrm{w} / \mathrm{w}$, which is around the same value of the difference between the weight loss of PP-1 and PP1/ CNT. As for the materials that were synthesized from polymerizations performed at shorter times (PP2/CNT and PP3/CNT), less weight loss was observed, indicating that the CNTs are incorporated into the polymer matrix in about $4 \% \mathrm{w} / \mathrm{w}$. The initial temperature of degradation $\left(\mathrm{T}_{\text {onset }}\right)$ and the maximum degradation temperature $\left(\mathrm{T}_{\max }\right)$ of the synthesized PP/CNT were shifted to higher values compared to those of PP-1, indicating there was an increase in thermal stability of the polymer matrix caused by adding the CNTs. Moreover, in the cases of PP2/CNT and PP3/CNT, a decrease of the difference between $\mathrm{T}_{\text {max }}$ and $\mathrm{T}_{\text {onset }}$ was observed, which is characteristic behavior of nanocomposites.

In Figure 2 the profiles of TGA curves related to the materials obtained are shown. There was a shift of the curves of PP/CNT nanocomposites to higher values of degradation temperature in relation to those corresponding

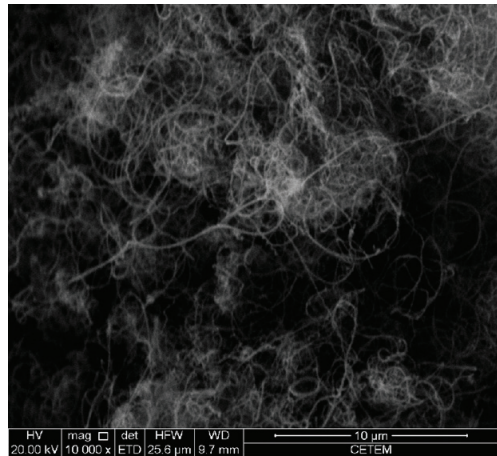

(a)

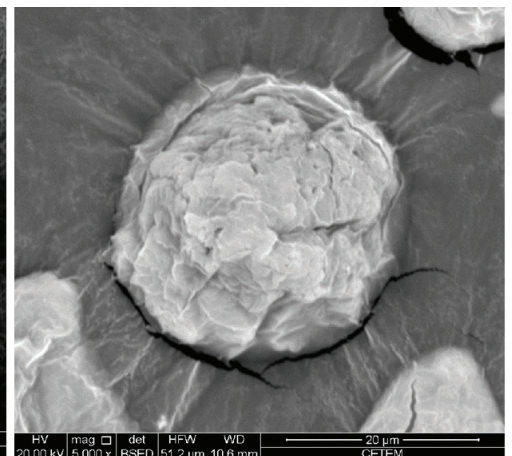

(b)

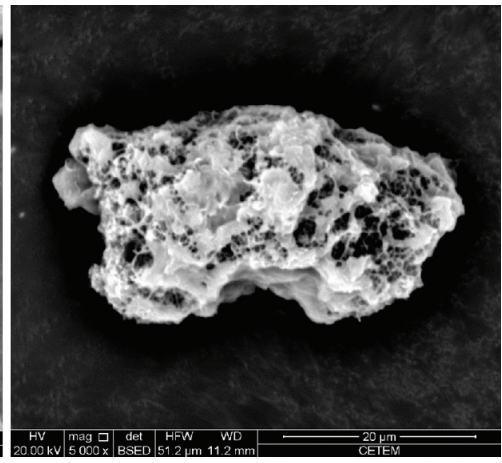

(c)

Figure 1. (a) Carbon nanotubes; (b) Standard Ziegler-Natta catalyst (CAT-1); (c) Ziegler-Natta catalyst with immobilized carbon nanotubes (CAT-CNT)

Table 1. Yield of reactions and thermal stability of polymers.

\begin{tabular}{lcccccc}
\hline \multicolumn{1}{c}{ Sample } & Reaction time (min) & Yield $(\mathbf{g}$ PP) & Weight loss $(\boldsymbol{\%})$ & $\mathbf{T}_{\text {onset }}\left({ }^{\circ} \mathbf{C}\right)$ & $\mathbf{T}_{\max }\left({ }^{\circ} \mathbf{C}\right)$ & $\mathbf{T}_{\max }-\mathbf{T}_{\text {onset }}\left({ }^{\circ} \mathbf{C}\right)$ \\
\hline PP-1 & 60 & 3.68 & 99.3 & 425 & 444 & 19 \\
PP1/CNT & 60 & 6.14 & 98.8 & 451 & 480 & 29 \\
PP2/CNT & 30 & 0.69 & 95.3 & 451 & 460 & 9 \\
PP3/CNT & 15 & 0.72 & 95.8 & 449 & 457 & 8 \\
\hline
\end{tabular}

PP-1 = neat polypropylene, originated from the standard catalyst; PP1/CNT, PP2/CNT, PP3/CNT = nanocomposites obtained at different polymerization times; $\mathrm{T}_{\text {onset }}=$ initial temperature of degradation; $\mathrm{T}_{\max }=$ temperature of maximum degradation rate. 
to the polymer without nanofiller PP-1. Note also that the composite PP1/CNT, containing around $0.5 \% \mathrm{CNT}$, presented higher thermal stability, probably due to the smaller quantity of CNT aggregates.

The polymer materials were pressed into DMA samples, which were cryofractured, and their surfaces were characterized by SEM. The micrographs are shown in Figure 3.

There was a very homogeneous distribution of the nanotubes throughout the matrix polymer in the nanocomposites. In PP1/CNT, the presence of isolated CNT could still be observed, despite the greater dilution of nanofiller in the polymer matrix. PP2/CNT showed good distribution, since it is possible to see isolated CNTs structures, and the natural nanotube tangles were eliminated. In turn, PP3/CNT showed concentrations of larger particles in some regions of the sample. This may be due to the short time of propylene polymerization.

Transmission electronic microscopy (TEM) was also used to observe the dispersion of nanofillers in the polymer matrix of the sample PP1/CNT (Figure 4). The micrographs revealed by TEM in various regions showed the same pattern, proving that it was possible to obtain a good dispersion of the CNTs in the PP matrix. The black dots present in the micrographs are related to the presence of original CNT catalyst residue.

The thermal properties of the materials obtained were evaluated by differential scanning calorimetry (DSC).

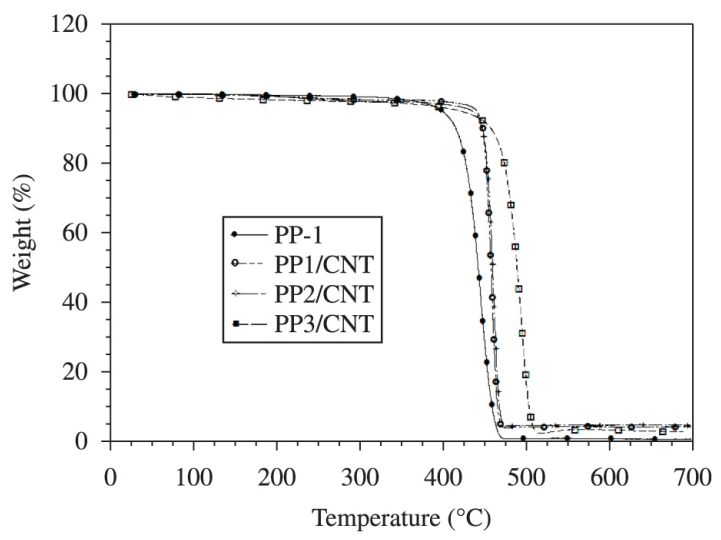

Figure 2. TGA profiles of the polymers.
The data obtained are listed in Table 2, as well as the results of the dynamic-mechanical analysis (DMA).

The presence of the CNTs in the polymerization influenced the thermal properties of the polymers, as revealed by the non-isothermal DSC experiments, since an increase of the nanocomposites' properties was found. There was a slight increase in the melting temperature $\left(\mathrm{T}_{\mathrm{m}}\right)$ of PP in the nanocomposites compared to the neat polymer. This can be attributed to the fact that the presence of CNTs reduced the mobility and melting entropy of PP, due to their constraint effect on the polymer segments and chains ${ }^{[27]}$. Also, it can be attributed to the effect of CNTs on the isospecific catalyst sites. The crystallization temperature $\left(\mathrm{T}_{\mathrm{c}}\right)$ of all synthesized nanocomposites showed a significant increase, although they contained different concentrations of CNT in the matrix. The degree of crystallinity $\left(\mathrm{X}_{c}\right)$ of the nanocomposites also showed a significant increase compared to the neat polymer, with that of PP2/CNT presenting the highest value, suggesting the action of CNT as a nucleating agent for PP. In addition to Table 2, Figure 5 shows DSC profiles, indicating a difference between the DSC curves of the nanocomposites compared to that of the neat polymer. This can be noted in the greatly enlarged endotherm of PP-1, suggesting the presence of PP crystals with different sizes and perfections, which may be due to the presence of polypropylene chains having different levels of isotacticity. This is typical behavior of polymers obtained with catalysts with multiple active sites, such as traditional Ziegler-Natta. However, polymers containing CNTs have narrower melting profiles, although bimodal, with higher content of more perfect crystals with thicker lamellae, since the melting peaks are shifted to higher temperatures.

This variation may be related to the nucleating effect of CNTs or even the influence on the nature of the catalytic sites by these nanofillers present in the catalyst particles. In fact, it seems that the number of less isospecific sites decreased, replaced by more isospecific ones, producing a polymer with higher fraction of PP increased $\mathrm{T}_{\mathrm{m}}$, which is the opposite of the profile of the PP-1 endotherm. On the other hand, according to reports in the literature ${ }^{[28]}$, narrower crystallization and melting peaks can suggest a narrower crystallite size distribution in MWNT/PP composites compared to pure PP. Higher

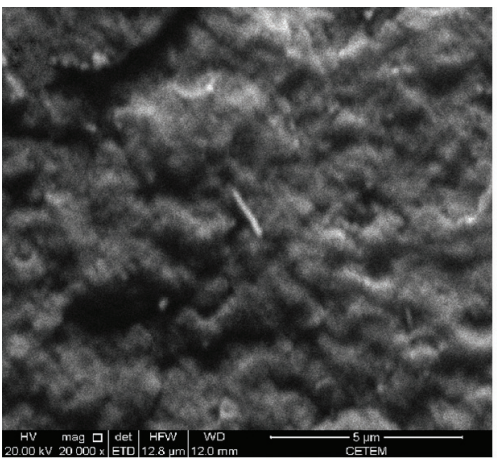

(a)

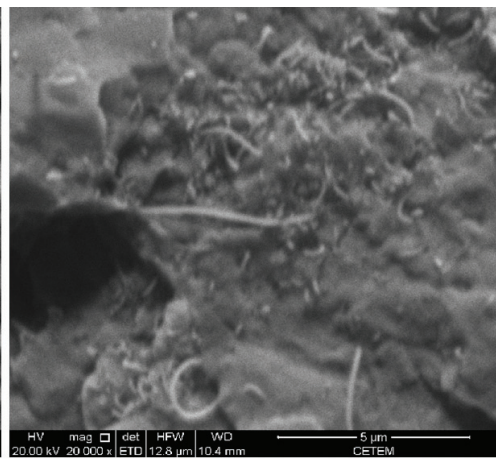

(b)

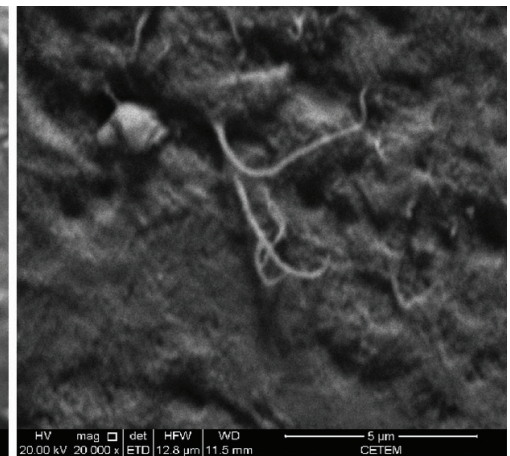

(c)

Figure 3. SEM of the nanocomposites. (a) PP1/CNT; (b) PP2/CNT; (c) PP3/CNT. 


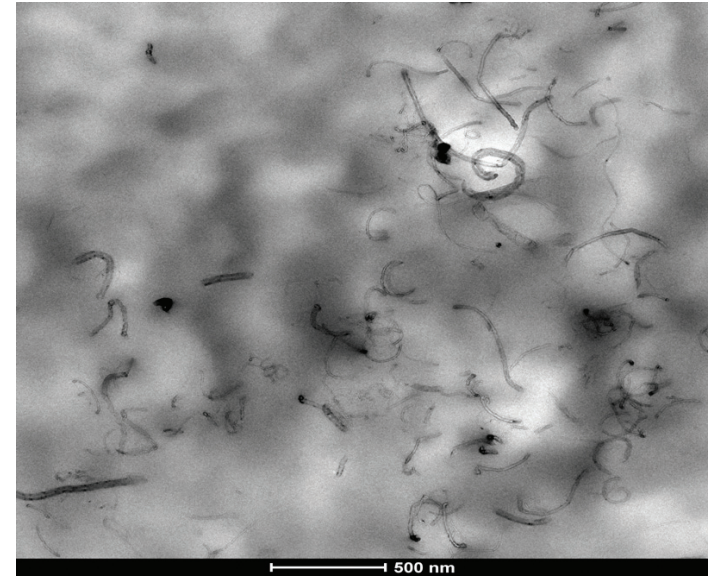

Figure 4. TEM of nanocomposite PP1/CNT.

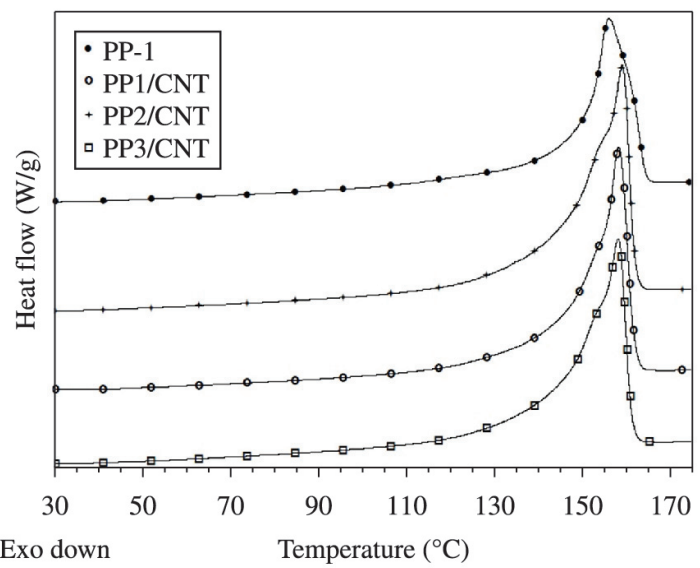

Figure 5. DSC profiles of the polymers obtained.

Table 2. Thermal properties and dynamic-mechanical analysis of polymers.

\begin{tabular}{lcccccc}
\hline \multirow{2}{*}{ Sample } & \multicolumn{3}{c}{ DSC } & \multicolumn{3}{c}{ DMA } \\
\cline { 2 - 7 } & $\mathbf{T}_{\mathbf{m}}\left({ }^{\circ} \mathbf{C}\right)$ & $\mathbf{T}_{\mathbf{c}}\left({ }^{\circ} \mathbf{C}\right)$ & $\mathbf{X}_{\mathbf{c}}(\boldsymbol{\%})$ & $\mathbf{E}^{\prime} \mathbf{2 5}^{\circ} \mathbf{C}(\mathbf{M P a})$ & $\mathbf{E} \mathbf{2 5}^{\circ} \mathbf{C}(\mathbf{M P a})$ & $\mathbf{T}_{\mathbf{g}}\left({ }^{\circ} \mathbf{C}\right)$ \\
\hline PP-1 & 156 & 116 & 35 & 1938 & 107 & 7.0 \\
PP1/CNT & 158 & 124 & 41 & 1953 & 107 & 8.4 \\
PP2/CNT & 159 & 125 & 47 & 2220 & 126 & 8.5 \\
PP3/CNT & 158 & 125 & 40 & 1958 & 125 & 6.3 \\
\hline
\end{tabular}

PP-1 = neat polypropylene, originated from the standard catalyst; PP1/CNT, PP2/CNT, PP3/CNT = nanocomposites obtained at different polymerization times; $\mathrm{T}_{\mathrm{m}}=$ melting temperature; $\mathrm{T}_{\mathrm{c}}=$ crystallization temperature; $\mathrm{X}_{\mathrm{c}}=$ degree of crystallinity; $\mathrm{E}^{\prime}=$ storage modulus; $\mathrm{E}^{\prime \prime}=$ loss modulus; $\mathrm{T}_{\mathrm{g}}=$ glass transition temperature.

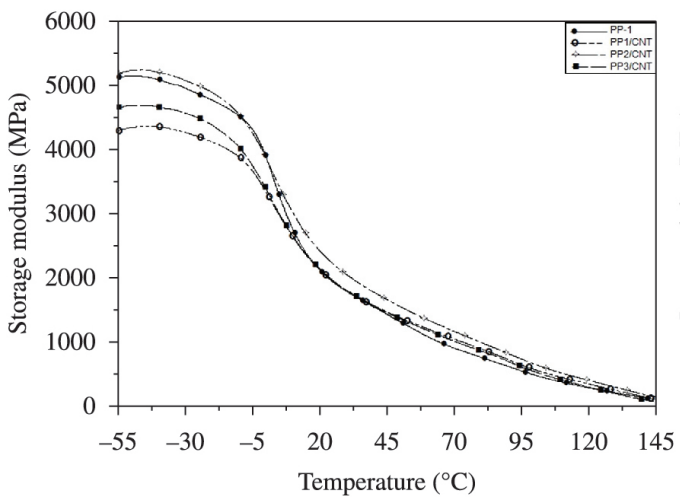

(a)

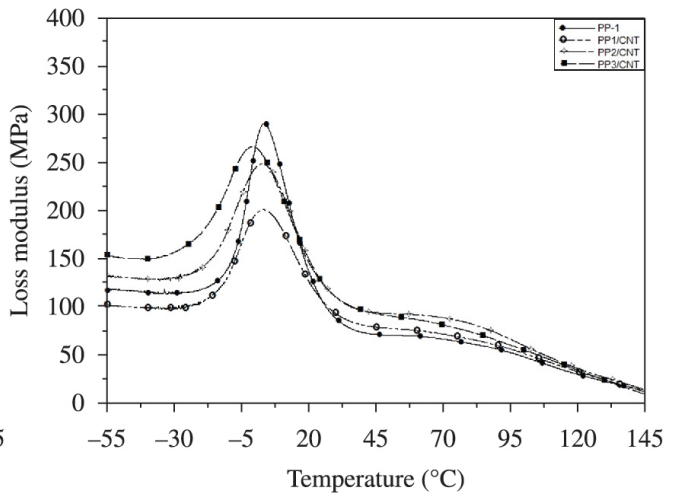

(b)

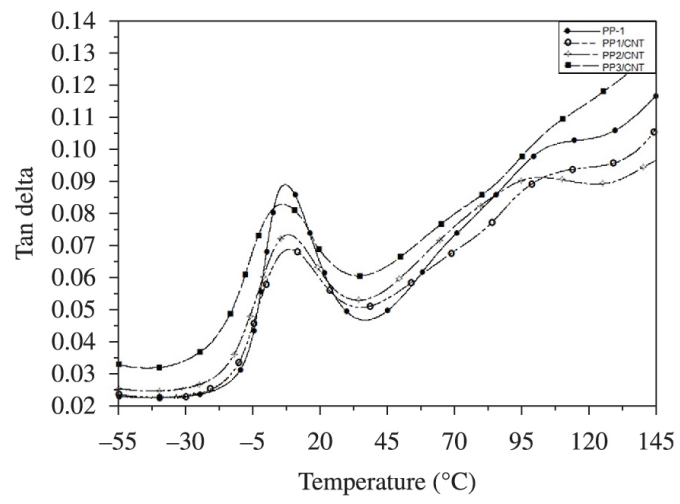

(c)

Figure 6. Storage modulus (a), loss modulus (b), and Tan Delta (c) as a function of temperature. 
thermal conductivity of the CNTs as compared to that of the polymer may be responsible, at least in part, for the sharper crystallization and melting peaks, as heat will be more evenly distributed in the samples containing the CNTs.

In Table 2 the dynamic-mechanical properties of the polymers synthesized from Ziegler-Natta catalysts are also listed, where the moduli E' and E" were compared at $25{ }^{\circ} \mathrm{C}$. PP $2 / \mathrm{CNT}$ had an increase of about $15 \%$ in storage modulus compared to PP-1, but the other nanocomposites did not show a substantial increase in E'. In relation to the loss modulus, both PP2/CNT and PP3/CNT showed an increase of about $17 \%$ while $\mathrm{PP} 1 / \mathrm{CNT}$ presented the same value as PP-1. The curves of storage modulus and loss modulus and the damping factor (tan delta) are shown in Figure 6.

The increase in E' for PP2/CNT, which means an increase in stiffness of the material, occurred throughout the temperature range studied. The DMA analysis was also used to determine the glass transition temperature $\left(\mathrm{T}_{\mathrm{g}}\right)$ of the polymers, whose values were determined from the Tan $\delta$ curves. The $\mathrm{T}_{\mathrm{g}} \mathrm{s}$ of PP1/CNT and PP2/CNT were shifted to appreciably higher values while the PP3/CNT decreased. In all materials, the damping factor decreased, showing increased rigidity of the polymer chains due to the presence of CNT.

\section{Conclusion}

CNT was successfully immobilized on ZieglerNatta catalysts based on titanium species supported on magnesium chloride, which enabled the preparation of PP/CNT nanocomposites with different percentages of nanofiller by the in situ polymerization technique. A homogeneous distribution of CNT was achieved and the activity of the catalyst with CNT was higher than that of the standard catalyst, which was directly related to the increased catalyst porosity due to the immobilized nanofiller.

By TGA it was possible to confirm that the nanotubes were incorporated into the PP matrix, increasing the initial temperature of degradation. Even in PP1/CNT, which showed no residual mass by TGA, showed an increase in initial temperature of degradation.

The micrographs revealed by SEM showed that the CNTs were dispersed in the polymer matrix, as confirmed by TEM micrographs, confirming that it is possible to obtain well dispersed CNTs in polymer matrixes.

Among the properties observed by DSC analysis, the degree of crystallinity and crystallization temperature presented the most significant effects. The nanotubes acted as nucleating agents and also increased the $\mathrm{T}_{\mathrm{c}}$. The tension tests performed by DMA showed considerable change only in PP2/CNT, in which both the storage and loss moduli increased, as well as the glass transition temperature of PP.

\section{Acknowledgements}

Project FP7-PEOPLE-IRSES-2011-295262 acronym VAIKUTUS

\section{References}

1. Ma, P. C.; Siddiqui, N. A.; Marom, G. \& Kim, J. K. - Compos. Part A, 41, p.1345 (2010). http://dx.doi. org/10.1016/j.compositesa.2010.07.003

2. Al Saleh, M. H. \& Sundararaj, U. - Carbon, 47, p.2 (2009). http://dx.doi.org/10.1016/j.carbon.2008.09.039

3. Thomassin, J. M.; Huynen, I.; Jerome, R. \& Detrembleur, C. - Polymer, 51, p.115 (2010). http://dx.doi.org/10.1016/j. polymer.2009.11.012

4. Zhou, X. W.; Zhu, Y. F. \& Liang, J. - Mater. Res. Bull., 42, p.456 (2007). http://dx.doi.org/10.1016/j. materresbull.2006.06.027

5. Hammel, E.; Tang, X.; Trampert, M.; Schmitt, T.; Mauthner, K.; Eder, A. \& Pötscherke, P. - Carbon, 42, p.1153 (2004). http://dx.doi.org/10.1016/j.carbon.2003.12.043

6. Keith, J. M.; King, J. A. \& Johnson, B. A. - J. New Mater. Electrochem. Syst., 11, p.253 (2008).

7. Uchida T.; Anderson, D. P.; Minus, M. L. \& Kuma, S. - J. Mater. Sci., 41, p.5851 (2006). http://dx.doi.org/10.1007/ s10853-006-0324-0

8. Li, J.; Wong, P. S. \& Kim, J. K. - Mater. Sci. Eng. A., 483, p.660 (2008). http://dx.doi.org/10.1016/j.msea.2006.08.145

9. Banerjee, B. S; Hemraj-Benny, T. \& Wong, S. S. - Adv. Mater., 17, p.17 (2005). http://dx.doi.org/10.1002/ adma. 200401340

10. Funck, A. \& Kaminsky, W. - Compos. Sci. Technol., 67, p.906 (2006). http://dx.doi.org/10.1016/j. compscitech.2006.01.034

11. Sahoo, N. G.; Rana, S.; Cho J. W., Li, L. \& Chan, S. H. - Prog. Polym. Sci., 35, p.837 (2010). http://dx.doi. org/10.1016/j.progpolymsci.2010.03.002

12. Andoni, A.; Chadwick, J. C.; Milani, S.; Niemantsyerdriet, H. J. W. \& Thüne, P. C. - J. Catal, 247, p.129 (2007). http:// dx.doi.org/10.1016/j.jcat.2007.01.019

13. Kaushik, V. K.; Gupta,V. K. \& Naik, D. G. XPS. - Appl. Surf. Sci., 253, p.753 (2006). http://dx.doi.org/10.1016/j. apsusc.2006.01.002

14. Huang, J. \& Rempel, G. L. - Prog. Polym. Sci., 20, p.459 (1995). http://dx.doi.org/10.1016/0079-6700(94)00039-5

15. Mukhopadhyay, S.; Kulkarni, S. A. \& Bhaduri, S. - J. Organomet. Chem., 690, p.1356 (2005). http://dx.doi. org/10.1016/j.jorganchem.2004.12.002

16. Stukalov, D. V.; Zakharov, V. A.; Potapov, A. G. \& Bukatov, G. D. - J. Catal., 266, p.39 (2009). http://dx.doi. org/10.1016/j.jcat.2009.05.012

17. Kissin, Y. V.; Liu, X.; Pollic,D. J.; Brungard, N. L. \& Chang, M. - J. Mol. Catal. A: Chem., 287, p.45 (2008). http://dx.doi.org/10.1016/j.molcata.2008.02.026

18. Oliveira, R. J. B.; Forrester, A. M. S. \& Marques, M. F. V. - Macromol. Symp., 299-300, p.215 (2011). http:// dx.doi.org/10.1002/masy.200900122

19. Oliveira, R. J. B. "Estabilização de polipropileno com antioxidantes naturais: comparação entre as técnicas de mistura in situ e no estado fundido", Dissertação de Mestrado, Universidade Federal do Rio de Janeiro, Brasil (2010).

20. Almeida, L. A. "Nanocompósitos de polietileno e polipropileno sintetizados com catalisadores Ziegler-Natta 
com morfologia controlada", Dissertação de Mestrado, Universidade Federal do Rio de Janeiro, Brasil (2008).

21. Marques, M. F. V.; Cardoso, R. S. \& Galvão, G. S. - Appl. Catal. A, 374, p.65 (2010). http://dx.doi.org/10.1016/j. apcata.2009.11.027

22. Cardoso, R. S. "Desenvolvimento de catalisadores Ziegler-Natta com morfologia controlada para síntese de polipropileno/argila”, Tese de Doutorado, Universidade Federal do Rio de Janeiro, Brasil (2011).

23. Akinci, A. - Arch. Mater. Sci. Eng., 35, p.91 (2009).

24. Chrissafis, K.; Paraskevopoulos, K. M.; Stavrev, S. Y.; Docoslis, A.; Vassiliou, A. \& Bikiaris, D. N. - Thermochim. Acta, 465, p.6 (2007). http://dx.doi.org/10.1016/j. tca.2007.08.007
25. Song, P.; Zhao, L.; Cao, Z. \& Fang, Z. - J. Mater. Chem., 21, p.7782 (2011). http://dx.doi.org/10.1039/c1jm10395d

26. Ganß, M.; Satapathy, B. K.; Thunga, M.; Weidisch, R.; Pötschke, P. \& Jehnichen, D. - Acta Mater., 56, p.2227 (2008).

27. Xia, H.; Wang, Q.; Li, K.; Hu, G.-H. - J. Appl. Polym. Sci., 93, p.378 (2004). http://dx.doi.org/10.1002/app.20435

28. Seo, M.-K.; Lee, J-R. \& Park, S.-J. - Mat. Sci. Eng. : A, 404, p.79 (2005). http://dx.doi.org/10.1016/j.msea.2005.05.065

Received: 01/30/13

Revised: 08/12/13

Accepted: 09/09/13 